\title{
Cardioprotective Role of Myocardial Endothelial Nitric Oxide Synthase and Serum Nitric Oxide Induced by Hexarelin in Rats with Myocardial Infarction- Induced Heart Failure
}

\author{
Elvis Agbo ${ }^{1,}$, Donghai Liu ${ }^{2}$, Jiawan Liao ${ }^{3}$, Roland Osei Saahene ${ }^{4}$, and Precious Barnes ${ }^{5}$ \\ ${ }^{1}$ Associate Professor, Department of Human Anatomy, Histology and Embryology, College of Medicine, Jinggangshan University, Ji'an City, Jiangxi Province, China \\ 2 MD, College of Medicine, Jiamusi University, Jiamusi City, Heilongjiang Province, China \\ 3 Professor, Department of Human Anatomy, Histology and Embryology, College of Medicine, Jinggangshan University, Ji'an City, Jiangxi Province, China \\ $4 \mathrm{PhD}$, Department of Microbiology and immunology, School of Medical Science, College of Health and Allied Sciences, University of Cape Coast, Ghana \\ 5 PhD, Department of Physician Assistant Studies, School of Allied Health Sciences, College of Health and Allied Sciences, University of Cape Coast, Ghana
}

* Corresponding author: Elvis Agbo, Department of Human Anatomy, Histology and Embryology, College of Medicine, Jinggangshan University, Ji'an City, Jiangxi Province, China. Tel: +8613904547645; Email: dr.elvis.agbo@gmail.com

Received 2021 April 05; Revised 2021 May 01; Accepted 2021 May 15.

\begin{abstract}
Background: Growth hormone-releasing peptides (GHRP) have been reported to possess cardioprotective properties; nonetheless, their mechanisms of action are still not very clear.

Objectives: Some studies have suggested that modulation of endothelial nitric oxide synthase (eNOS) and the upregulation of nitric oxide (NO) are cardioprotective. Therefore, the present study strived to test the hypothesis that a potent GHRP analog (hexarelin) could increase serum nitric oxide level and regulate myocardial eNOS to alleviate the development of heart failure.

Methods: Myocardial infarction-induced heart failure in rats was established by permanent coronary artery ligation. The sham group, control group, and heart failure group all received normal saline (100 $\mu \mathrm{g} / \mathrm{kg}$; SC BID; 30days), while the rats in the hexarelin treatment group were treated with hexarelin (100 $\mu \mathrm{g} / \mathrm{kg}$, SC BID, 30 days). The rats were tested for myocardial apoptosis, oxidative stress, left ventricular function, various molecular analyses, as well as pathological and structural myocardial changes.

Results: Hexarelin treatment improved contractile function and attenuated myocardial histopathological damages, oxidative stress, fibrosis, as well as apoptosis. All these were accompanied by the upregulation of myocardial eNOS and an increase in serum NO concentration.

Conclusion: As evidenced by the obtained results, the anti-cardiac failure capacity of hexarelin in a rat model is mediated by an increase in serum nitric oxide level and the up-modulation of myocardial eNOS; therefore, they can be considered therapeutic targets against heart failure.
\end{abstract}

Keywords: Apoptosis, Heart failure, Hexarelin, eNOS, Myocardial infarction, Nitric oxide

\section{Background}

Hexarelin is a hexapeptide belonging to a family of non-natural peptides known as growth hormonereleasing peptides (GHRP) (1). Their numerous beneficial cardiovascular functions are mediated by intracellular mechanisms and specific receptors quite different from those of growth hormone-releasing hormones (GHRH) (1). The exertion of those reported cardioprotective effects by hexarelin is achieved by binding to either cardiac non-growth hormone secretagogue receptor CD36 or the growth hormone secretagogue receptor-1a (2). Some of the beneficial cardiovascular functions of hexarelin include its antiatherosclerotic functions (3), suppression of cardiac fibrosis $(4,5)$, anti-apoptotic effects $(6,7)$, and cardiac output improvement (8-10).

Myocardial infarction (MI) is still the commonest etiology of heart failure in our world today, while heart failure continues to be the determining factor in poor prognosis following a MI over the past five decades (11). Since decades of promotion of myocardial repair has been an unsuccessful therapy in the fight against heart failure, there is a critical need for more different and diverse approaches.

Various studies suggested that nitric oxide (NO) and endothelial nitric oxide synthase (eNOS) perform vital roles in cardiovascular health. For instance, reduced availability of NO is influential in controlling pathophysiological processes, such as myocardial dysfunction and remodeling, which can lead to or worsen heart failure in patients suffering from MI (12) or in experiments involving myocardial ischemia (13). The NO is widely known as a rapid and reversible inhibitor of mitochondrial respiration and oxygen consumption by tissues. Moreover, when the production of NO is inhibited, it leads to impaired endothelial-dependent vasodilation, augmentation of cardiac remodeling (14), a reduction in the neovascularization of the myocardium, as well as the impairment of cardiac efficiency and perfusion (15).

On the other hand, eNOS is involved in processes related to the regulation of the supply and consumption of oxygen in the microcirculation of skeletal and cardiac muscles (14). It is capable of stimulating angiogenesis and increasing myocardial perfusion via vasodilation; therefore, it can be very beneficial post-MI (16). In 2001, the experimental

Copyright (c) 2021, Author(s). This is an open-access article distributed under the terms of the Creative Commons Attribution-NonCommercial 4.0 International License (http://creativecommons.org/licenses/by-nc/4.0/) which permits copy and redistribute the material just in noncommercial usages, provided the original work is properly cited 
findings of a study conducted by Scherrer-Crosbie et al. indicated that there was a less pronounced myocardial remodeling and left ventricular dysfunction in the wild-type mice, as compared to much deterioration in myocardial remodeling and left ventricular dysfunction in eNOS-deficient mice after MI (17). Although hexarelin is a potential therapeutic agent against some experimental cardiovascular conditions, its mechanisms of action are still not clear.

\section{Objectives}

Hexarelin administration has once been demonstrated to exhibit some cardioprotective properties against atherosclerosis in a rat model through the modulation of aortic eNOS and serum NO (3). Therefore, the current study strived to test the hypothesis that hexarelin could regulate serum NO level and myocardial eNOS to ameliorate myocardial infarction-induced heart failure in rats.

\section{Methods}

\subsection{Animals and Drugs}

The procedures used in the current study were all approved and performed in line with the guidelines for care and use of laboratory animals, published by the Ministry of Science and Technology of the People's Republic of China and the Animal Research Ethics Committee of Jinggangshan University. For the purpose of the study, 98 10-12 week-old male Sprague-Dawley (SD) rats (205-225g) were bought from Nanchang University's Laboratory Animal Centre, China. Under a 12-h light/dark cycle, the rats were kept in a temperature-controlled room $\left(22 \pm 2^{\circ} \mathrm{C}\right)$ and got free access to water and food. Hexarelin was obtained from ProSpec (NJ, East Brunswick, USA) and dissolved in saline to the required concentration prior to its administration in our experiment.

\subsection{Myocardial infarction-induced heart failure model preparation and experimental protocols}

Randomly selected SD rats formed the control group $(n=19)$, while the other rats went through coronary artery ligation surgery. The rat models of heart failure were established by the ligation of the left anterior descending coronary artery as demonstrated in previous literature (18). In brief, after anesthetizing SD rats with an intraperitoneal injection (ip) of $10 \%$ chloral hydrate $(0.3 \mathrm{~mL} / 100 \mathrm{~g})$, the ventilation of rats was achieved using a constantvolume rodent ventilator. The thorax of rats was surgically opened, and permanent ligation of the left anterior descending coronary artery was carried out using a silk suture (7-0) at about $0.3 \mathrm{~cm}$ from its origin. The presence of an ischemic regional color change and an ST-segment elevation on an electrocardiogram (ECG) confirmed a successful coronary artery ligation surgical procedure.

Before the closure of muscles and skin, the heart was placed in the thoracic cavity and manual air evacuation was performed. The randomly selected SD rats that formed the sham group $(n=19)$ went through a similar surgical procedure with the exception of artery ligations. Four weeks postsurgery, ligated rats that survived underwent a random selection and formed the heart failure group $(\mathrm{HF} ; \mathrm{n}=18)$ and hexarelin treatment group ( $\mathrm{HF}+\mathrm{Hx}$; $n=18)$. Rats in every group underwent a 30-day treatment as follows: $\mathrm{HF}+\mathrm{Hx}$ rats had hexarelin (100 $\mu \mathrm{g} / \mathrm{kg}$, twice a day) subcutaneous injection (19), while all rats in the other groups were treated using an equal volume of saline.

\subsection{Echocardiography}

Upon the completion of 30-day treatment of all rats, echocardiography was performed on them by a professional echocardiographer blinded to all the rat groups. Rats were anesthetized using $10 \%$ chloral hydrate $(0.3 \mathrm{~mL} / 100 \mathrm{~g}$, ip), and M-mode twodimensional stable images of the parasternal shortand long-axes views were recorded with Philips Sonos 7500 echocardiography system using a 12$\mathrm{MHz}$ transducer. All measured parameters were averaged from three consecutive cardiac cycles: the left ventricular end-systolic diameter (LVESD), the left ventricular end-diastolic diameter (LVEDD), and a real-time calculation of left ventricular ejection fraction (LVEF).

\subsection{Tissue preparation}

After taking all the echocardiographic measurements, the hearts of the rats were rapidly harvested. Tissues in non-infarcted left ventricular regions were cut at the papillary muscle level in cross-section. A portion of tissues was frozen using liquid nitrogen, stored at $-80^{\circ} \mathrm{C}$, and later used for reverse-transcription quantitative real-time polymerase chain reaction (RT-qPCR) and western blotting. Other portions were directly fixed in $10 \%$ phosphate-buffered formalin, dehydrated, and embedded in paraffin. Rat blood samples were centrifuged at $1500 \mathrm{~g}$ for $10 \mathrm{~min}$ at $4^{\circ} \mathrm{C}$ and then stored at $-80^{\circ} \mathrm{C}$.

\subsection{Hematoxylin and eosin (H\&E) staining of cardiac tissues}

Some $4-\mu \mathrm{m}$ slices of tissue samples embedded with paraffin wax were heated using an incubator and underwent dewaxing and H\&E staining. Sample slices from each group were assessed by a pathologist blinded to all the rat groups and scored them with respect to the following parameters: acute cell death or myocardial necrosis, degeneration of cardiac muscles, neutrophil infiltration, and hemorrhage. 
3.6. Hoechst 33258 staining for assessment of cell apoptosis

Dewaxed 4- $\mu$ m slices of tissue samples embedded with paraffin wax underwent staining with Hoechst 33258 stain in cautious adherence to the manufacturer's manual. Slide observation was performed with an Olympus fluorescence microscope, and Image-Pro Plus (version 6.0) was used for the evaluation of apoptosis.

\subsection{Masson's trichrome staining for the assessment of cardiac fibrosis \\ $4-\mu \mathrm{m}$ sections of paraffin wax-embedded tissues were heated using a $60^{\circ} \mathrm{C}$ incubator and underwent dewaxing and then Masson's trichrome dye staining. The images of heart sections were then captured with an Olympus DM4000 light microscope. The interstitial fibrosis area of the left ventricle (Interstitial fibrotic area of $\mathrm{LV}=$ collagen area in $\mathrm{LV} /$ total LV area) was evaluated with an imaging software (Image-Pro Plus 6.0).}

\subsection{Assessment of myocardial malondialdehyde (MDA)} level and superoxide dismutase (SOD) activity level

After preparing homogenates from rat cardiac muscle tissue samples, levels of myocardial malondialdehyde (MDA) and superoxide dismutase (SOD) activity were assessed with kits from Nanjing Jiancheng Institute of Bioengineering and strict adherence to the manufacturer's instructions.

\subsection{Immunohistochemistry}

Myocardial tissue samples were prepared for immunohistochemical analysis of caspase-3, Bcl-2, and Bax proteins. In summary, 4- $\mu \mathrm{m}$ tissue sections fixed with formalin and embedded with paraffin wax underwent deparaffinization and rehydration before rinsing three times in phosphate-buffered saline (PBS). The activities of endogenous peroxidases were blocked by a 15 -min incubation at $37^{\circ} \mathrm{C}$ with $3 \%$ hydrogen peroxide before being washed three times in PBS. Incubation of sections at $37^{\circ} \mathrm{C}$ for $30 \mathrm{~min}$ in goat serum was conducted for blockage of nonspecific binding, followed by washing three times in PBS.

The antibodies used in the incubation of tissue sections at $37^{\circ} \mathrm{C}$ for $30 \mathrm{~min}$ were as follows: Caspase-3 (1:50; 19677-1-AP) and anti-Bax (1:20; 50599-2-Ig) antibodies bought from Proteintech Co. (IL, Chicago, USA), and anti-Bcl-2 antibody (1:50; AF6139) obtained from Affinity Biosciences (Cambridge, UK). Thereafter, washing was performed using PBS. Sections were incubated for 20 min using a biotinylated goat anti-rabbit secondary antibody and for $15 \mathrm{~min}$ at $37^{\circ} \mathrm{C}$ using a conjugated working solution of streptavidinhorseradish peroxidase (HRP), followed by washing again in PBS. The reactions were developed by incubating with 3, 3'-diaminobenzene (DAB) for 5 min and counterstaining with Mayer's hematoxylin for $30 \mathrm{sec}$. The slides were mounted under a light microscope after rinsing and dehydration. Density analysis of the images was performed using imaging software (Image-Pro Plus 6.0).

\subsection{Reverse-transcription quantitative real-time polymerase chain reaction (RT-qPCR)}

TRIzol reagent was used in extracting total RNA from cardiac tissues before the utilization of the Prime Script Rt Reagent Kit, supplied by TakaraChina for their reverse transcription into cDNA by strictly following the manufacturer's manual. The following primers obtained from Sangon Biological Engineering Technology Co. (Shanghai-China) were used: $\beta$-actin, forward: 5'-GAGGCTCTCTTCCAG CCTTC-3', reverse: 5'- AGGGTGTAAAA-CGCAGCTCA3'; eNOS, forward: 5'-ATGGCGAAGCGTGTGAAG-3', reverse: 5'- ATTGTGGCTCGGGTGGAT-3'; procollagenI, forward: 5'-TGCCGTGACCT-CAAGATGTG-3', reverse: 5'-CACAAGCGTGCTGTAGGTGA-3'; iNOS, forward: 5'AGAAGTCCAGCCGCACCAC-3', reverse: 5'-TGGTTGC CTGGGAAAATCC-3' and TGF $\beta-1$, forward: 5'AAGAAGTCACCCGCGTGCTA-3', reverse: 5'-TGTGTGATGTCTTTGGTTTTGTCA-3'. SYBR Premix Ex Taq (Takara Bio Inc) was used in the RT-qPCR quantification of gene expression. The normalization of interested gene expressions was performed with $\beta$-actin expression serving as the control. The $2-\Delta \Delta C t$ method was used for the calculation of relative gene expression.

\subsection{Western blot analysis}

Myocardial tissue-extracted proteins separated in SDS-poly-acrylamide gel were transferred electrophoretically onto a poly-vinylidene fluoride membrane, blocked using nonfat milk (5\%), and underwent an overnight incubation at a temperature of $4^{\circ} \mathrm{C}$ with primary-antibodies for cleaved caspase-3 (9664; 1:1000) obtained from Cell Signalling Technology (Danvers, USA), BNP (DF6902; 1:500) bought from Affinity Biosciences (Cambridge, UK), and $\beta$-Actin (AF0003; 1:1000) bought from Beyotime (Jiangsu, China). Finally, after incubation with HRPconjugated secondary-antibody, immuno-reactive bands were detected using a chemiluminescence kit.

\subsection{Serum nitrite level determination by spectrophotometry}

The primary oxidation products of $\mathrm{NO}$ are nitrite and nitrate due to the reaction of NO with oxygen; therefore, the total concentration of serum nitrite and nitrate was used as a biomarker of total NO synthesized. A series of reactions involving the initial enzymatic reduction of serum nitrate to nitrite before measuring total serum nitrite level as an indicator of NO synthesis was achieved by using NO kit purchased from JingmeiBioTech Co. Ltd, Beijing, China, in close adherence to the manufacturer's protocol. Total 
serum nitrite concentration for each sample was evaluated by measuring the optical density of each sample at $530 \mathrm{~nm}$ (OD530) and comparing it with the OD530 of a standard nitrite calibration curve.

\subsection{Statistical analysis}

Experimental data were expressed as mean \pm standard deviation (SD). Statistical analysis was performed in SPSS software (version 24.0). Assessment of the statistical significance of the differences between the various groups was achieved by using one-way-analysis-of-variance (ANOVA) or Welch's method, followed by LSD post-hoc test when appropriate. A p-value less than 0.05 was considered statistically significant.

\section{Results}

4.1. Hexarelin improves left ventricular function in MIinduced heart failure rats

There was a significantly increased LVEDD and LVESD $(\mathrm{P}<0.05)$ with a significantly decreased $(\mathrm{P}<$ 0.05 ) LVEF in rats in the HF group (Figure 1a, 1b, and $1 \mathrm{c})$, compared to the Ctrl group rats, indicating diastolic and systolic function impairment, as well as a decline in cardiac contractile function. Meanwhile, $\mathrm{HF}$ rats treated with hexarelin had a significant decrease in LVESD and LVEDD $(\mathrm{P}<0.05)$ with a significantly $(\mathrm{P}<0.05)$ elevated LVEF (Figure $1 \mathrm{a}, 1 \mathrm{~b}$, and $1 \mathrm{c}$ ), in comparison with the rats in the HF group.
This finding signified that hexarelin attenuated myocardial remodeling and improved myocardial contractile function in MI-induced cardiac failure rats.

4.2. Effect of Hexarelin on the levels of myocardial malondialdehyde and superoxide dismutase activity

As displayed in Figure $1 \mathrm{~d}$ and 1e, SOD activity significantly decreased $(\mathrm{P}<0.05)$, while MDA content significantly increased $(\mathrm{P}<0.05)$ in the HF group, compared with that in the Ctrl group. Hexarelin administration yielded opposite results by significantly $(\mathrm{P}<0.05)$ reversing of down-regulation and up-regulation of SOD activity and MDA content, respectively.

\subsection{Hexarelin attenuated myocardial pathological and structural changes in MI-induced heart failure rats}

As displayed in Figure 2a, compared to the Ctrl group, the myocardial tissues of HF group rats had severe pathological changes and structural damages, such as the rupturing and fragmentation of the myocardium, the loss of cross striations of myocardial fibers, loss of myocardial cells, as well as severe inflammatory cell infiltration. There was a clear improvement or attenuation in all the aforementioned myocardial pathological and structural changes as observed in hexarelin treated rats, compared with $\mathrm{HF}$ rats without hexarelin treatment.
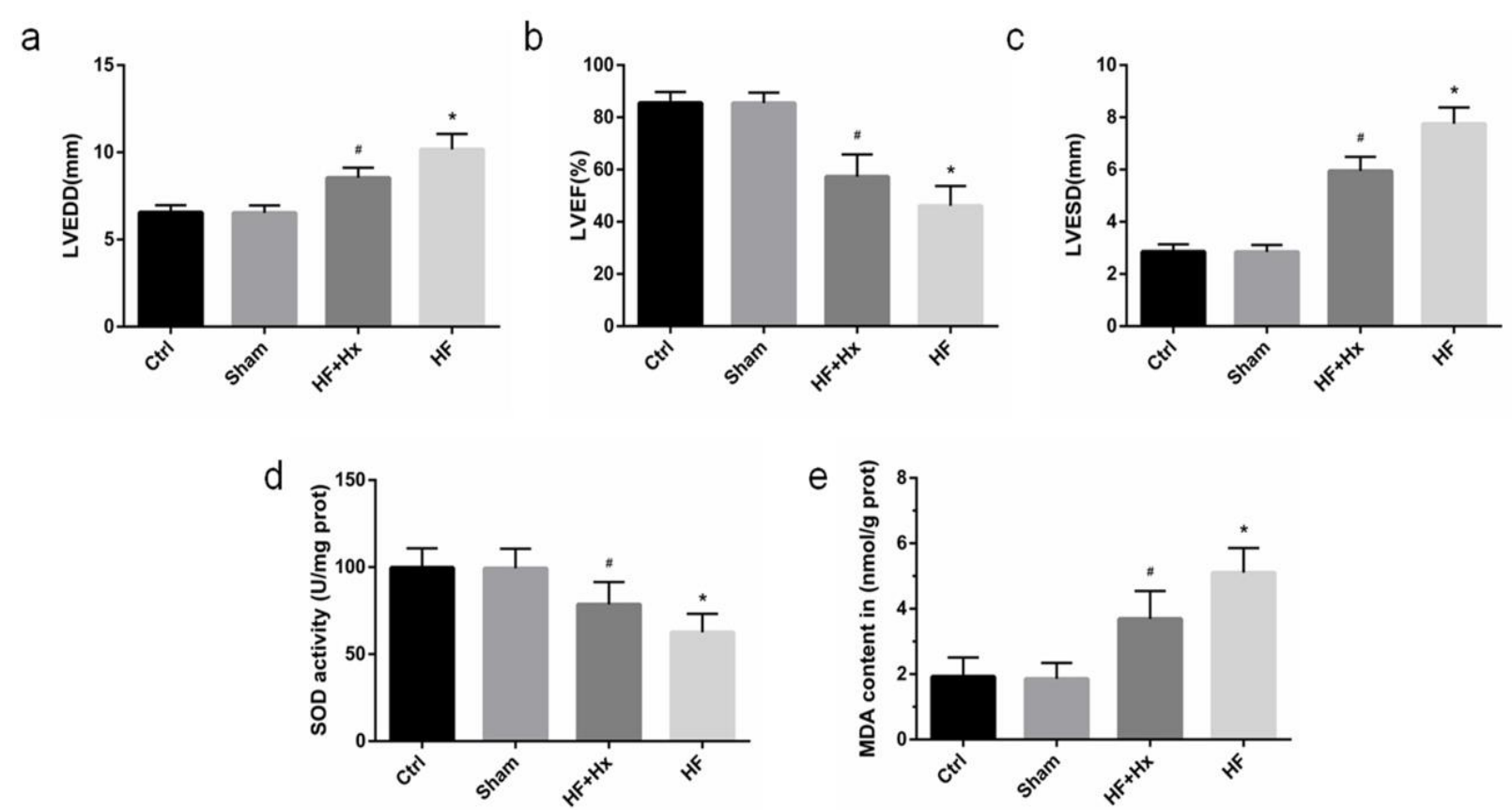

Figure 1. Results of echocardiography (a, b, c) demonstrating the effect of hexarelin on Myocardial infarction (MI)-induced heart failure (HF) rats with regards to: (a) left ventricular end-systolic diameter, (b) left ventricular ejection fraction, and (c) left ventricular end-diastolic diameter, Anti-oxidative effects of Hexarelin on MI-induced HF rats concerning (d) superoxide dismutase (SOD) and (e) malondialdehyde (MDA), All data were expressed as mean $\pm \mathrm{SD}(\mathrm{n}=6) .{ }^{*} \mathrm{P}<0.05$ in comparison with the control group; $\# \mathrm{P}<0.05$ compared to the HF group 
4.4. Amelioration of myocardial fibrosis and collagen deposition by hexarelin in MI-induced heart failure rats

The images of Masson's trichrome-stained cardiac sections are depicted in Figure $2 \mathrm{~b}$ with blue and red colors, indicating collagen fibers and normal cardiac tissues, respectively. Compared to the Ctrl group, collagen deposition was markedly elevated in $\mathrm{HF}$ group rats $(\mathrm{P}<0.05)$; however, it was significantly attenuated in $\mathrm{HF}$ rats treated with hexarelin, compared to that in HF group rats without hexarelin treatment. Moreover, $\mathrm{LV}$ interstitial fibrosis area $(\mathrm{P}<$ $0.05)$, which is a measure of interstitial fibrosis levels in various groups as shown in Figure 2c, confirmed and showed a trend very similar to that of collagen deposition described above.

\subsection{Hexarelin down-regulates cardiac fibrosis-related biomarkers in MI-induced heart failure rats}

RT-qPCR results (Figure $2 \mathrm{~d}$ and $2 \mathrm{e}$ ) indicated that procollagen-I and TGF- $\beta 1$ mRNA expressions in the myocardia of HF group rats were markedly $(\mathrm{P}<0.05)$ up-regulated, in comparison with that of the Ctrl group rats, pointing to a sharp rise in myocardial fibrosis level of the HF group. Nevertheless, it was realized that there was a significant drop in the levels of procollagen-I and TGF- $\beta 1$ gene expressions in the myocardia of $\mathrm{HF}$ rats treated with hexarelin, compared to that in HF group rats without hexarelin treatment $(\mathrm{P}<0.05)$.

\subsection{Hoechst Dye Staining and immunohistochemical assessment of apoptosis-related biomarkers}

As presented in Figure 3a and $3 \mathrm{~b}$, the population of cardiomyocyte nuclei showing apoptotic features, such as condensation and induced fragmentation of cardiomyocyte nuclei revealed as a bright fluorescence signal, increased significantly in HF rats, in comparison with Ctrl group rats $(\mathrm{P}<0.05)$. Nonetheless, these apoptotic features were markedly reduced in $\mathrm{HF}+\mathrm{Hx}$ rats, in comparison with $\mathrm{HF}$ rats $(\mathrm{P}<0.05)$. Our immunohistochemistry results (Figure $4 \mathrm{a}$ and $4 \mathrm{~d}$ ) suggested that Bax protein expression level in HF group rats increased significantly $(\mathrm{P}<0.05)$, compared to the Ctrl group.

However, treatment with hexarelin markedly $(\mathrm{P}<0.05)$ inhibited the rise in Bax protein expression level by comparing the $\mathrm{HF}+\mathrm{Hx}$ group with the HF rats. On the contrary, Bcl-2 protein expression level (Figure $4 \mathrm{~b}$ and $4 \mathrm{e}$ ) in $\mathrm{HF}$ rats significantly reduced, in comparison with the Ctrl group $(\mathrm{P}<0.05)$. Nonetheless, hexarelin treated rats had a significant up-regulation in Bcl-2 expression, compared to the HF rats without hexarelin treatment $(\mathrm{P}<0.05)$. In addition, a capase- 3 protein expression level (Figure $4 \mathrm{c}$ and $4 \mathrm{f}$ ) showed a similar trend with that of Bax protein expression.

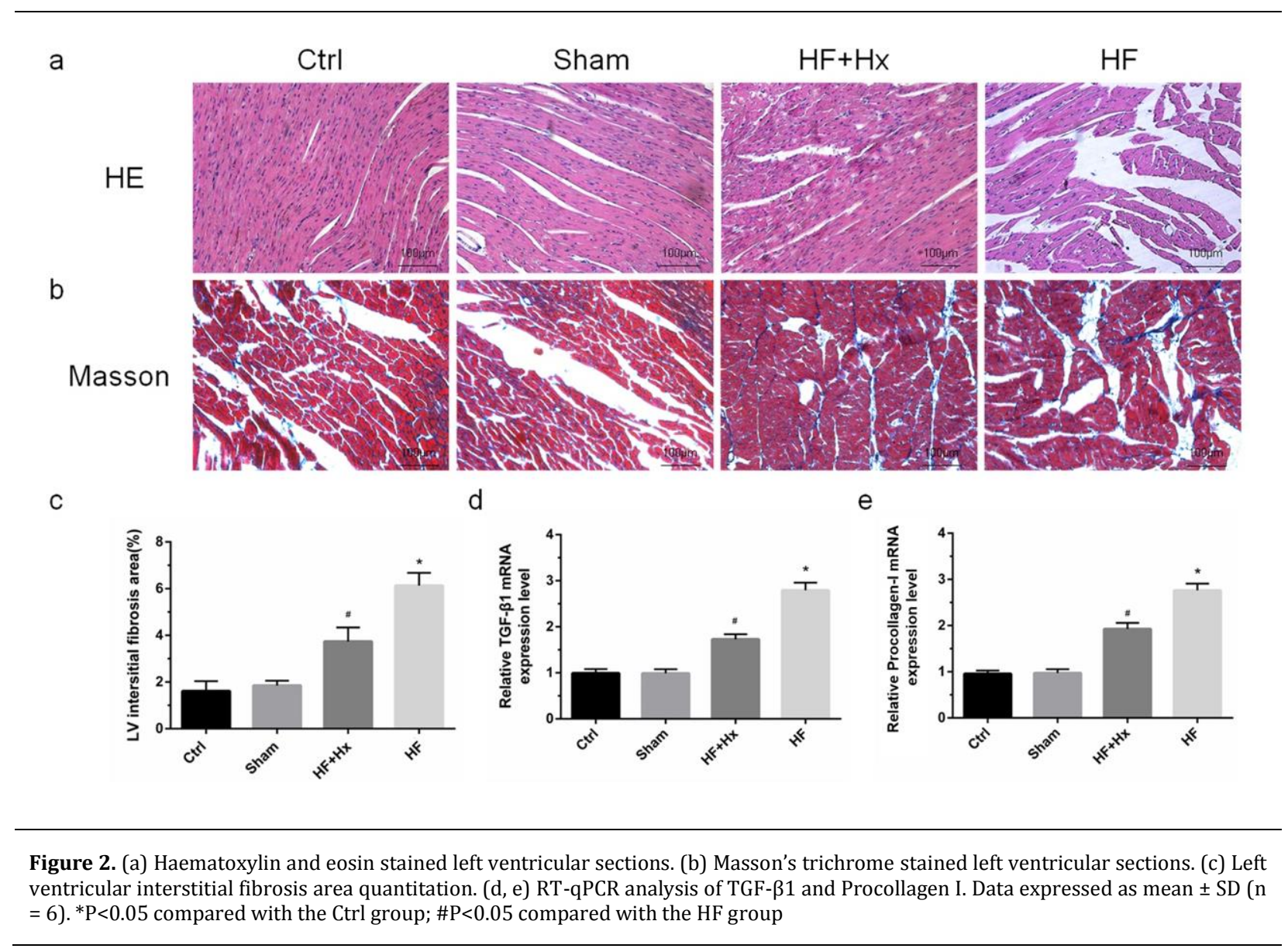


a
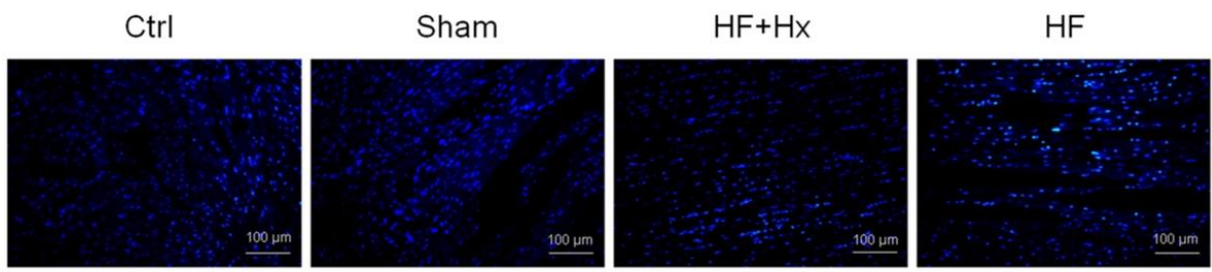

$\mathrm{b}$

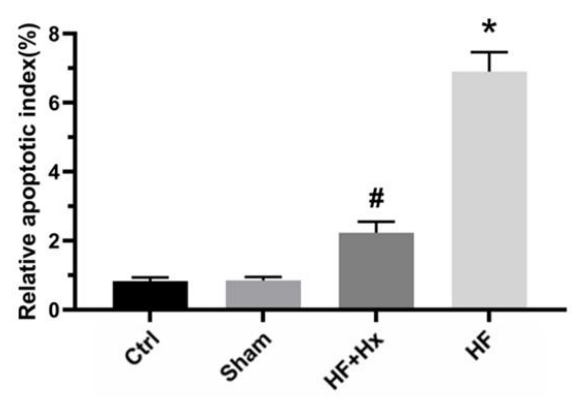

Figure 3. (a) Hoechst stained left ventricular sections showing hexarelin's anti-apoptotic effects. (b) Relative apoptotic index. All data expressed as mean \pm SD $(n=6)$. ${ }^{*} \mathrm{P}<0.05$ compared with the Ctrl group; $\# \mathrm{P}<0.05$ compared with the HF group

4.7. Western blot results of brain natriuretic peptide and Cleaved caspase-3 proteins

As illustrated in Figure 5a, b, and c, the levels of brain natriuretic peptide (BNP) and cleaved caspase-3 protein expressions which are myocardial remodeling and apoptosis indicators, respectively,

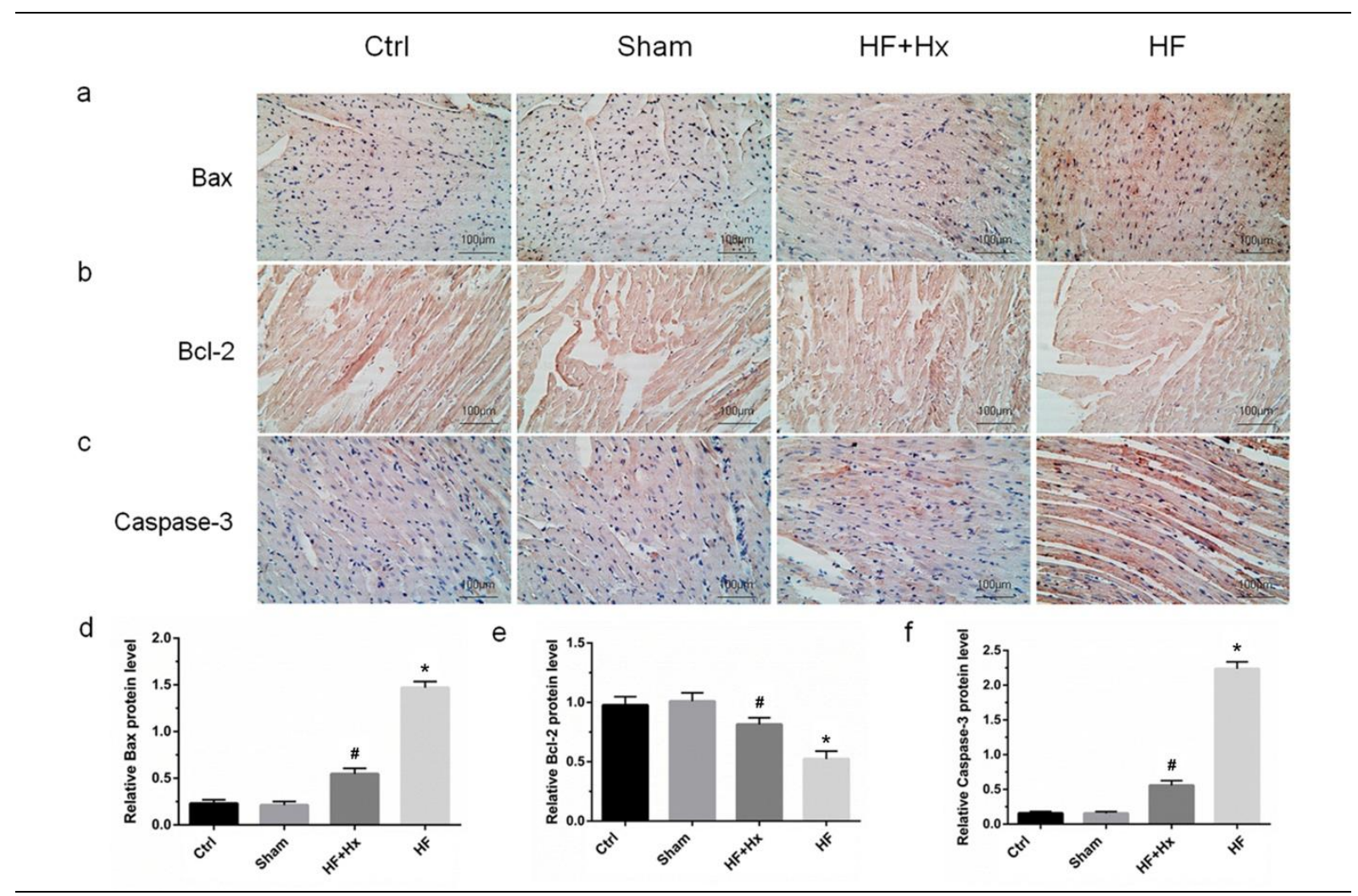

Figure 4. (a, d) Immunohistochemistry results and relative integral optical density (IOD) quantitative analysis of Bax. (b, e) Immunohistochemistry results and relative IOD quantitative analysis of Bcl-2. (c, f) Immunohistochemistry results and relative IOD quantitative analysis of Caspase-3. All data expressed as mean $\pm \mathrm{SD}(\mathrm{n}=6)$. ${ }^{*} \mathrm{P}<0.05$ compared with the Ctrl group; \#P<0.05 compared with the HF group 


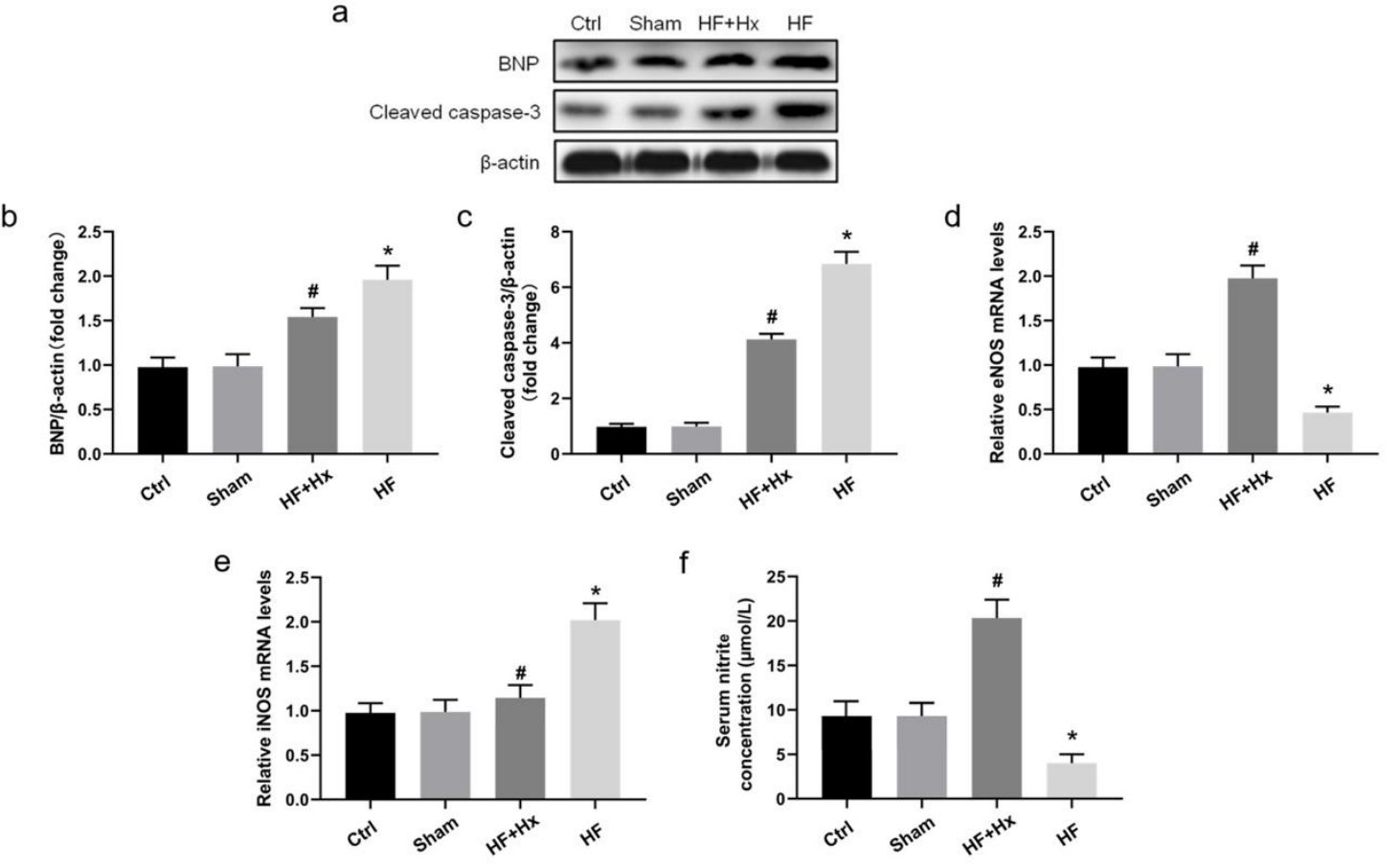

Figure 5. (a, b, c) Representative western blot and quantitative analysis of BNP and Cleaved caspase-3. (d, e) RT-qPCR analyses of eNOS and iNOS. (f) Serum nitrite concentrations in the Ctrl, Sham, HF+Hx and HF groups. All data expressed as mean \pm SD ( $=6$ ). $* \mathrm{P}<0.05$ compared with the Ctrl group; \# $\mathrm{P}<0.05$ compared with the HF group

were all significantly elevated in the HF group, in comparison with those in Ctrl group rats $(\mathrm{P}<0.05)$. There was a marked decrease $(\mathrm{P}<0.05)$ in BNP and cleaved caspase- 3 levels in the myocardia of HF rats treated with hexarelin, compared to that in HF group rats without hexarelin treatment.

\subsection{Effect of hexarelin on myocardial eNOS and iNOS in MI-induced heart failure rats}

Based on the results of RT-qPCR (Figure $5 \mathrm{~d}$ and 5e), eNOS mRNA and iNOS mRNA expressions in HF group rats significantly decreased and increased, respectively $(\mathrm{P}<0.05)$, in comparison with the Ctrl and sham groups. Nonetheless, there was a significant elevation of eNOS gene expression and a significant decrease in iNOS gene expression in the myocardia of $\mathrm{HF}$ rats treated with hexarelin, compared to that in HF group rats without hexarelin treatment $(\mathrm{P}<0.05)$.

\subsection{Effect of hexarelin on serum NO in MI-induced heart failure rats}

Serum nitrite level, which is a reflection of total serum NO synthesized, was investigated in the present study. The obtained results (Figure 5f) indicated that serum nitrite concentration in $\mathrm{HF}$ group rats decreased significantly $(\mathrm{P}<0.05)$, in comparison with the Ctrl and sham groups. However, there was a significant elevation of serum nitrite concentration in HF rats treated with hexarelin, compared to that in HF group rats without hexarelin treatment $(\mathrm{P}<0.05)$.

\section{Discussion}

The results of the present research strongly indicated that the enhancement of myocardial eNOS and serum NO mediated by hexarelin played a beneficial role in significant improvements in cardiac function and other positive cardioprotective properties against oxidative stress, apoptosis, cardiac hypertrophy, and cardiac fibrosis in the setting of MIinduced heart failure. This study also suggested that the attenuation of eNOS mRNA and NO bioavailability, accompanied by iNOS mRNA upregulation, contributed to cardiac dysfunction and cardiac failure progression in rats induced by coronary artery ligation.

The presence of eNOS in the cardiomyocyte and endothelium of the heart is known to be an important regulator of NO since it is mainly involved in the constitutive production of NO by its catalytic action on substrate L-arginine. The NO is a molecule serving in protection against numerous pathological cardiovascular conditions (20-22). For instance, a deficiency in vascular NO level is well known to cause endothelial dysfunction (23) which is a pathological condition strongly associated with post-MI-induced heart failure $(24,25)$.

Consistent with the results of the present 
research, numerous studies provided evidence concerning a severe reduction in NO bioavailability in patients with heart failure $(13,26-28)$ and experimental MI conditions $(12,29,30)$. Moreover, various mechanisms have been reported to contribute to the pathophysiological etiology of heart failure due to attenuated eNOS and NO levels, and a conspicuous factor among them is oxidative stress. Reduced NO bioavailability observed in heart failure is accompanied by an elevation in reactive oxygen species, such as superoxide anion, and a depleted antioxidant defensive reserve in the heart $(30,31)$, leading to oxidative stress injury. Multiple studies have pointed out that NO is capable of abrogating calcium overload and oxidative injury in the mitochondrion, as well as attenuating superoxide anion synthesis (32-34). Therefore, the preservation of NO levels, together with its eNOS regulator, is vital in the protection of the heart after ischemic conditions, such as MI. In addition, unchecked production of reactive oxygen species due to the lack of adequate antioxidant reserves, such as superoxide dismutase (SOD) enzymes promotes NO scavenging and the consequent reduction in its bioavailability (30). It explains why the reduction of SOD activity in MI-induced heart failure group rats was accompanied by decreased serum NO levels and an increase in the level of MDA which is a lipid peroxidation marker (35) and evidence of myocardial damage caused by oxidative stress in rats with heart failure. Pang et al. demonstrated that hexarelin exerts cardioprotective properties against atherosclerosis which is another cause of heart failure post-MI by the upregulation of eNOS mRNA and serum NO (3). The findings of the current study also indicated that hexarelin is capable of enhancing the bioavailability of eNOS and NO to ameliorate cardiac oxidative stress by both upregulating myocardial eNOS and inhibiting the possible inactivation of NO by radicals.

The cellular components of the heart, such as cardiomyocytes, endothelia, and myocardial microvasculature smooth muscles, all express iNOS in response to inflammatory cytokines $(36,37)$. Moreover, oxidative stress post-MI has been reported to be involved in the stimulation of iNOS production (38). Several reports indicated that heart failure patients show elevated iNOS in their myocardium (39-41). In line with this background, myocardial iNOS was significantly increased in post-MI-induced heart failure rats in the present study. Inflammatory cytokines are upregulated in rats with MI (42) or heart failure patients $(43,44)$. Since the results of the current study indicated oxidative stress injury and H\&E results demonstrated evidence of increased inflammation in MI-induced heart failure rats, inflammatory cytokines and oxidative stress could be possible causes of myocardial iNOS elevation in the present study.

Several pathophysiological events leading to cardiac dysfunction and remodeling take place after MI-induced heart failure which further deteriorates to cause death (45-48). Feng et al. proved that increased myocardial iNOS mRNA level post-MI leads to cardiac contractile function impairment or dysfunction, resulting in heart failure (49). A study carried out by Scherrer-Crosbie et al. used coronary artery ligation-induced MI in mice to investigate the involvement of eNOS in cardiac remodeling and LV dysfunction. In their experiment, there was a marked $\mathrm{LV}$ remodeling and dysfunction in eNOS-deficient mice, compared to normal control mice (17).

To buttress the role of eNOS in cardiac dysfunction and remodeling, Jones et al. also demonstrated that the overexpression of eNOS results in the attenuation of $\mathrm{LV}$ dysfunction to ameliorate heart failure in mice (50). Furthermore, NO is involved in the modulation of events that contribute to LV performance (51-53) and inhibition of excessive cardiomyocyte hypertrophy $(54,55)$.In agreement with the aforementioned studies, myocardial iNOS was increased, while myocardial eNOS and serum NO levels were significantly decreased in heart failure rats in the present study, leading to significant diastolic and systolic function impairment, as well as a decline in cardiac contractile function.

Increases in LVEDD, LVESD, and myocardial BNP protein levels in rats with heart failure all indicated cardiac enlargement or hypertrophic remodeling. It has been reported that hexarelin is capable of improving cardiac contractile function in infarcted hearts of rats (56) and inhibiting cardiac hypertrophy (7). Moreover, the results of the current research pointed out that hexarelin is cardioprotective by upregulating eNOS (3), as well as due to its antioxidative and anti-inflammatory properties. Therefore, hexarelin is capable of improving cardiac contractile function and inhibiting cardiac hypertrophic remodeling by the upregulation of myocardial eNOS and serum NO, as well as the inhibition of myocardial iNOS as confirmed by the results of the present study. Since hexarelin treatment of rats with heart failure increased serum NO levels, accompanied by an elevation in myocardial eNOS expression and a decrease in iNOS expression, it can be concluded that hexarelin may upregulate NO bioavailability by eNOS upmodulation.

Cardiac fibrosis, characterized by an excessive cardiac interstitial extracellular matrix (ECM) protein accumulation, is strongly related to pathological cardiac remodeling which progresses to cause heart failure $(57,58)$. An increase in ECM is caused by several factors, such as TGF- $\beta 1$, which stimulate cardiac and renal fibrosis (59). Procollagen is a precursor of collagen, a major ECM component of the myocardium; moreover, it has been implicated in the etiology of cardiac fibrosis and heart failure (60-62). Various reports demonstrated that NO limits the 
myocardial deposition of ECM to attenuate cardiac fibrosis $(54,55,63)$. Moreover, eNOS genes have been found to be anti-fibrotic in rats with MI (64). In the present study, it was observed that hexarelin exhibited anti-cardiac fibrotic properties by limiting the degree of interstitial fibrotic areas of myocardium, accompanied by a reduction in the mRNA of TGF- $\beta 1$ and procollagen-1. These results agree with the notion that the upregulation of eNOS and NO as observed in the present study in response to hexarelin treatment, limits cardiac fibrosis and, therefore, ameliorates heart failure.

Earlier reports have indicated that eNOS-derived NO inhibits cardiomyocyte apoptosis, playing an important role in protection against heart failure $(65,66)$. For instance, Smith et al. proved that apoptosis and necrosis are inhibited after incorporating a human eNOS gene into cardiac muscles of rats in an experimental MI study (65). Furthermore, since eNOS or eNOS-derived NO is involved in the stimulation of angiogenesis in ischemic tissues $(16,67)$, it might help in compensating for a high demand for oxygen post-MI to limit cardiac cell death. The eNOS upregulation also induces apoptosis as reported by $\mathrm{Hu}$ et al. who demonstrated that cardiomyocyte apoptosis associated with iNOS induction in heart failure rats was reversed by selective inhibition of iNOS expression (68).

In addition, the interaction between $\mathrm{NO}$ and antiapoptotic factor Bcl-2 or pro-apoptotic factors, such as caspase- 3 and Bax, has been elucidated as an explanation to the apoptotic-inhibitory effects of NO $(69,70)$. In the present study, immunohistochemical and Western Blot results showed that a decrease in anti-apoptotic Bcl-2, as well as an increase in proapoptotic caspase- 3 and Bax expressions, in rats with heart failure was substantially reversed by hexarelin administration. These findings were also consistent with the H\&E and Hoechst staining results of the present study.

In addition, even though hexarelin has been shown to be anti-apoptotic in some cardiovascular conditions $(6,7,71)$, the results of the current research shed some new light on this notion pointing to the association of the anti-apoptotic effect of hexarelin with the upregulation of eNOS expression or NO bioavailability and the inhibition of iNOS expression in MI-induced heart failure rat models. To even provide more mechanistic insights into eNOS/NO-mediated cardioprotection by hexarelin in MI-induced heart failure rats, the use of genetic ablation of eNOS genes can be incorporated in future studies to further investigate whether all these cardioprotective properties of hexarelin will be abrogated. Furthermore, although the present study was designed to assess whether myocardial eNOS and serum NO could be regulated by hexarelin to exhibit cardioprotective effects against failing hearts in rats, it is believed the quality of data can be improved in future studies by designing investigations revealing more explicit mechanisms of how hexarelin stimulates eNOS/NO production.

\section{Conclusion}

As evidenced by the results of the present study, the anti-cardiac failure capacity of hexarelin through its preservation of cardiac function, as well as its antioxidative, anti-apoptotic, and anti-cardiac remodeling properties, in MI-induced heart failure rat model is mediated by the up-modulation of myocardial eNOS expression and serum NO bioavailability; therefore, they can be considered therapeutic targets against heart failure.

\section{Footnotes}

Authors' Contribution: The current research was designed by Elvis Agbo and performed by Dong Hai Liu, Elvis Agbo, and Roland-Osei Saahene. The data was analyzed by Precious Barnes and Jia Wan Liao and the preparation of the article was done by Elvis Agbo.

Conflicts of Interest: The authors declare that they have no conflict of interest.

Ethical Approval: The procedures used in the current study were all performed in line with the guidelines for care and use of laboratory animals, published by the Ministry of Science and Technology of the People's Republic of China, as well as The Animal Research Ethics Committee of Jinggangshan University.

Funding/Support: The present research was supported by the doctoral Start-up Fund of the Natural Science Foundation of Jinggangshan University, China (JZB1925).

\section{References}

1. Ghigo E, Arvat E, Muccioli G, Camanni F. Growth hormonereleasing peptides. Eur J Endocrinol. 1997;136(5):445-60. doi: 10.1530/eje.0.1360445. [PubMed: 9186261].

2. Mao Y, Tokudome T, Kishimoto I, Otani K, Hosoda H, Nagai C, et al. Hexarelin treatment in male ghrelin knockout mice after myocardial infarction. Endocrinology. 2013;154(10):3847-54. doi: 10.1210/en.2013-1291. [PubMed: 23861368].

3. Pang J, Xu Q, Xu X, Yin H, Xu R, Guo S, et al. Hexarelin suppresses high lipid diet and vitamin D3-induced atherosclerosis in the rat. Peptides. 2010;31(4):630-8. doi: 10.1016/j.peptides.2009.11.007. [PubMed: 19931584].

4. Xu X, Pang J, Yin H, Li M, Hao W, Chen C, et al. Hexarelin suppresses cardiac fibroblast proliferation and collagen synthesis in rat. Am J Physiol Heart Circ Physiol. 2007;293(5):H2952-8. doi: 10.1152/ajpheart.00004.2007. [PubMed: 17766487].

5. Agbo E, Liu D, Li M, Saahene RO, Chen L, Zhao L, et al. Modulation of PTEN by hexarelin attenuates coronary artery ligation-induced heart failure in rats. Turk J Med Sci. 2019;49(3):945-58. doi: 10.3906/sag-1812-49. [PubMed: 31091855].

6. Filigheddu N, Fubini $A$, Baldanzi G, Cutrupi S, Ghè C, Catapano 
F, et al. Hexarelin protects H9c2 cardiomyocytes from doxorubicin-induced cell death. Endocrine. 2001;14(1):113-9. doi: 10.1385/END0:14:1:113. [PubMed: 11322493].

7. Agbo E, Li M, Wang Y, Saahene R, Massaro J, Tian G. Hexarelin protects cardiac $\mathrm{H} 9 \mathrm{C} 2$ cells from angiotensin II-induced hypertrophy via the regulation of autophagy. Pharmazie. 2019;74(8):485-91. doi: 10.1691/ph.2019.9324. [PubMed: 31526442].

8. Broglio F, Guarracino F, Benso A, Gottero C, Prodam F, Granata $\mathrm{R}$, et al. Effects of acute hexarelin administration on cardiac performance in patients with coronary artery disease during by-pass surgery. Eur J Pharmacol. 2002;448(2):193-200. doi: 10.1016/s0014-2999(02)01934-9. [PubMed: 12144941].

9. Sun Q, Ma Y, Zhang L, Zhao YF, Zang WJ, Chen C. Effects of GH secretagogues on contractility and $\mathrm{Ca} 2+$ homeostasis of isolated adult rat ventricular myocytes. Endocrinology. 2010;151(9):4446-54. doi: 10.1210/en.2009-1432. [PubMed: 20610573].

10. Tivesten As, Bollano E, Caidahl K, Kujacic V, Sun XY, Hedner T, et al. The growth hormone secretagogue hexarelin improves cardiac function in rats after experimental myocardial infarction. Endocrinology. 2000;141(1):60-6. doi: 10.1210/ endo.141.1.7249. [PubMed: 10614623].

11. Cahill TJ, Kharbanda RK. Heart failure after myocardial infarction in the era of primary percutaneous coronary intervention: mechanisms, incidence and identification of patients at risk. World J Cardiol. 2017;9(5):407-15. doi: 10.4330/wjc.v9.i5.407. [PubMed: 28603587].

12. Wiemer G, Itter G, Malinski T, Linz W. Decreased nitric oxide availability in normotensive and hypertensive rats with failing hearts after myocardial infarction. Hypertension. 2001; 38(6):1367-71. doi: 10.1161/hy1101.096115. [PubMed: 11751719].

13. Drexler H. Endothelium as a therapeutic target in heart failure. Circulation. 1998;98(24):2652-5. doi: 10.1161/01.cir.98.24. 2652. [PubMed: 9851948].

14. Cabrales P, Tsai AG, Frangos JA, Intaglietta M. Role of endothelial nitric oxide in microvascular oxygen delivery and consumption. Free Radic Biol Med. 2005;39(9):1229-37. doi: 10.1016/j.freeradbiomed.2005.06.019. [PubMed: 16214038].

15. Trochu JN, Bouhour JB, Kaley G, Hintze TH. Role of endothelium-derived nitric oxide in the regulation of cardiac oxygen metabolism: implications in health and disease. Circ Res. 2000;87(12):1108-17. doi: 10.1161/01.res.87.12.1108. [PubMed: 11110767].

16. Duda DG, Fukumura D, Jain RK. Role of eNOS in neovascularization: NO for endothelial progenitor cells. Trends Mol Med. 2004;10(4):143-5. doi: 10.1016/j.molmed.2004.02.001. [PubMed: 15162796].

17. Scherrer-Crosbie M, Ullrich R, Bloch KD, Nakajima H, Nasseri B, Aretz HT, et al. Endothelial nitric oxide synthase limits left ventricular remodeling after myocardial infarction in mice. Circulation. 2001;104(11):1286-91. doi: 10.1161/hc3601. 094298. [PubMed: 11551881].

18. Lygate C. Surgical models of hypertrophy and heart failure: Myocardial infarction and transverse aortic constriction. Drug Discov Today Dis Models. 2006;3(3):283-90. doi: 10.1016/j.ddmod.2006.10.002.

19. Xu X, Ding F, Pang J, Gao X, Xu RK, Hao W, et al. Chronic administration of hexarelin attenuates cardiac fibrosis in the spontaneously hypertensive rat. Am J Physiol Heart Circ Physiol. 2012;303(6):H703-11. doi: 10.1152/ajpheart.00257.2011. [PubMed: 22842067].

20. Furchgott RF, Zawadzki JV. The obligatory role of endothelial cells in the relaxation of arterial smooth muscle by acetylcholine. Nature. 1980;288(5789):373-6. doi: 10.1038/288373a0. [PubMed: 6253831].

21. Kubes P, Suzuki M, Granger D. Nitric oxide: an endogenous modulator of leukocyte adhesion. Proc Natl Acad Sci USA. 1991;88(11):4651-5. doi: 10.1073/pnas.88.11.4651. [PubMed: 1675786].

22. Kelly RA, Balligand JL, Smith TW. Nitric oxide and cardiac function. Circ Res. 1996;79(3):363-80. doi: 10.1161/01.res.79.3.363. [PubMed: 8781470].
23. Atochin DN, Huang PL. Endothelial nitric oxide synthase transgenic models of endothelial dysfunction. Pflug Arch Eur J Phy. 2010;460(6):965-74. doi: 10.1007/s00424-010-0867-4. [PubMed: 20697735].

24. Bauersachs J, Fleming I, Fraccarollo D, Busse R, Ertl G. Prevention of endothelial dysfunction in heart failure by vitamin E: attenuation of vascular superoxide anion formation and increase in soluble guanylyl cyclase expression. Cardiovasc Res. 2001;51(2):344-50. doi: 10.1016/s0008-6363(01)003194. [PubMed: 11470474].

25. Sartório CL, Fraccarollo D, Galuppo P, Leutke M, Ertl G, Stefanon I, et al. Mineralocorticoid receptor blockade improves vasomotor dysfunction and vascular oxidative stress early after myocardial infarction. Hypertension. 2007;50(5):919-25. doi: 10.1161/HYPERTENSIONAHA.107.093450. [PubMed: 17846350].

26. Treasure CB, Vita JA, Cox DA, Fish RD, Gordon JB, Mudge GH, et al. Endothelium-dependent dilation of the coronary microvasculature is impaired in dilated cardiomyopathy. Circulation. 1990;81(3):772-9. doi: 10.1161/01.cir.81.3.772. [PubMed: 2306829].

27. Hornig B, Maier V, Drexler H. Physical training improves endothelial function in patients with chronic heart failure. Circulation. 1996;93(2):210-4. doi: 10.1161/01.cir.93.2.210. [PubMed: 8548890].

28. Mohri M, Egashira K, Tagawa T, Kuga T, Tagawa H, Harasawa Y, et al. Basal release of nitric oxide is decreased in the coronary circulation in patients with heart failure. Hypertension. 1997;30(1):50-6. doi: 10.1161/01.hyp.30.1.50. [PubMed: 9231820].

29. Qi XL, Stewart DJ, Gosselin H, Azad A, Picard P, Andries L, et al. Improvement of endocardial and vascular endothelial function on myocardial performance by captopril treatment in postinfarct rat hearts. Circulation. 1999;100(12):1338-45. doi: 10.1161/01.cir.100.12.1338. [PubMed: 10491380].

30. Bauersachs J, Bouloumié A, Fraccarollo D, Hu K, Busse R, Ertl G. Endothelial dysfunction in chronic myocardial infarction despite increased vascular endothelial nitric oxide synthase and soluble guanylate cyclase expression: role of enhanced vascular superoxide production. Circulation. 1999;100(3):2928. doi: 10.1161/01.cir.100.3.292. [PubMed: 10411855].

31. Shiomi T, Tsutsui H, Matsusaka H, Murakami K, Hayashidani S, Ikeuchi $\mathrm{M}$, et al. Overexpression of glutathione peroxidase prevents left ventricular remodeling and failure after myocardial infarction in mice. Circulation. 2004;109(4):544-9. doi: 10.1161/01.CIR.0000109701.77059.E9. [PubMed: 14744974].

32. Gao L, Yin H, Smith RS, Chao L, Chao J. Role of kallistatin in prevention of cardiac remodeling after chronic myocardial infarction. Lab Invest. 2008;88(11):1157-66. doi: 10.1038/ labinvest.2008.85. [PubMed: 18762777].

33. Rakhit RD, Mojet MH, Marber MS, Duchen MR. Mitochondria as targets for nitric oxide-induced protection during simulated ischemia and reoxygenation in isolated neonatal cardiomyocytes. Circulation. 2001;103(21):2617-23. doi: 10.1161/01.cir.103.21.2617. [PubMed: 11382733].

34. Rastaldo R, Cappello S, Folino A, Berta GN, Sprio AE, Losano G, et al. Apelin-13 limits infarct size and improves cardiac postischemic mechanical recovery only if given after ischemia. Am J Physiol Heart Circ Physiol. 2011;300(6):H2308-15. doi: 10.1152/ajpheart.01177.2010. [PubMed: 21378145].

35. Azizi Y, Faghihi M, Imani A, Roghani M, Nazari A. Post-infarct treatment with [Pyr1]-apelin-13 reduces myocardial damage through reduction of oxidative injury and nitric oxide enhancement in the rat model of myocardial infarction. Peptides. 2013;46:76-82. doi: 10.1016/j.peptides.2013.05.006. [PubMed: 23727032].

36. Balligand JL, Ungureanu D, Kelly RA, Kobzik L, Pimental D, Michel T, et al. Abnormal contractile function due to induction of nitric oxide synthesis in rat cardiac myocytes follows exposure to activated macrophage-conditioned medium. J Clin Invest. 1993;91(5):2314-9. doi: 10.1172/JCI116461. [PubMed: 8486792].

37. Brady A, Poole-Wilson PA, Harding SE, Warren JB. Nitric oxide production within cardiac myocytes reduces their contractility 
in endotoxemia. Am J Physiol. 1992;263(6 Pt 2):H1963-6. doi: 10.1152/ajpheart.1992.263.6.H1963. [PubMed: 1481919].

38. Kobayashi N, Yoshida K, Nakano S, Ohno T, Honda T, Tsubokou Y, et al. Cardioprotective mechanisms of eplerenone on cardiac performance and remodeling in failing rat hearts. Hypertension. 2006;47(4):671-9. doi: 10.1161/01.HYP.0000203148.42892.7a. [PubMed: 16505212].

39. Haywood GA, Tsao PS, Von Der Leyen HE, Mann MJ, Keeling PJ, Trindade PT, et al. Expression of inducible nitric oxide synthase in human heart failure. Circulation. 1996;93(6):108794. doi: 10.1161/01.cir.93.6.1087. [PubMed: 8653828].

40. Drexler H, Kästner S, Strobel A, Studer R, Brodde OE, Hasenfuss G. Expression, activity and functional significance of inducible nitric oxide synthase in the failing human heart. J Am Coll Cardiol. 1998;32(4):955-63. doi: 10.1016/s0735-1097(98)00336-2. [PubMed: 9768717].

41. Vejlstrup NG, Bouloumie A, Boesgaard S, Andersen CB, NielsenKudsk JE, Mortensen SA, et al. Inducible nitric oxide synthase (iNOS) in the human heart: expression and localization in congestive heart failure. J Mol Cell Cardiol. 1998;30(6):121523. doi: 10.1006/jmcc.1998.0686. [PubMed: 9689595].

42. Ikeda U, Murakami Y, Kanbe T, Shimada K. $\alpha$-Adrenergic stimulation enhances inducible nitric oxide synthase expression in rat cardiac myocytes. J Mol Cell Cardiol. 1996;28(7):1539-45. doi: 10.1006/jmcc.1996.0144. [PubMed: 8841941].

43. Habib FM, Springall DR, Davies GJ, Oakley CM, Polak J, Yacoub M. Tumour necrosis factor and inducible nitric oxide synthase in dilated cardiomyopathy. Lancet. 1996;347(9009):1151-5. doi: 10.1016/s0140-6736(96)90610-8. [PubMed: 8609750].

44. Levine B, Kalman J, Mayer L, Fillit HM, Packer M. Elevated circulating levels of tumor necrosis factor in severe chronic heart failure. $N$ Engl J Med. 1990;323(4):236-41. doi: 10.1056/NEJM199007263230405. [PubMed: 2195340].

45. Boersma E, Mercado N, Poldermans D, Gardien M, Vos J, Simoons ML. Acute myocardial infarction. Lancet. 2003; 361(9360):847-58. doi: 10.1016/S0140-6736(03)12712-2. [PubMed: 12642064].

46. Jugdutt BI. Ventricular remodeling after infarction and the extracellular collagen matrix: when is enough enough? Circulation. 2003;108(11):1395-403. doi: 10.1161/01.CIR. 0000085658.98621.49. [PubMed: 12975244].

47. Kumar D, Jugdutt BI. Apoptosis and oxidants in the heart. $J$ Lab Clin Med. 2003;142(5):288-97. doi: 10.1016/S00222143(03)00148-3. [PubMed: 14647032].

48. Melo LG, Pachori AS, Kong D, Gnecchi M, Wang K, Pratt RE, et al. Molecular and cell-based therapies for protection, rescue, and repair of ischemic myocardium: reasons for cautious optimism. Circulation. 2004;109(20):2386-93. doi: 10.1161/01.CIR.0000128597.37025.00. [PubMed: 15159329].

49. Feng Q, Lu X, Jones DL, Shen J, Arnold JM. Increased inducible nitric oxide synthase expression contributes to myocardial dysfunction and higher mortality after myocardial infarction in mice. Circulation. 2001;104(6):700-4. doi: 10.1161/hc3201. 092284. [PubMed: 11489778].

50. Jones SP, Greer JJ, Van-Haperen R, Duncker DJ, de Crom R, Lefer DJ. Endothelial nitric oxide synthase overexpression attenuates congestive heart failure in mice. Proc Natl Acad Sci USA. 2003;100(8):4891-6. doi: 10.1073/pnas.0837428100. [PubMed: 1267698].

51. Kupatt C, Hinkel R, Vachenauer R, Horstkotte J, Raake P, Sandner T, et al. VEGF165 transfection decreases postischemic NF-KB-dependent myocardial reperfusion injury in vivo: role of eNOS phosphorylation. FASEB J. 2003;17(6):705-7. doi: 10.1096/fj.02-0673fje. [PubMed: 12586740].

52. Gyurko R, Kuhlencordt P, Fishman MC, Huang PL. Modulation of mouse cardiac function in vivo by eNOS and ANP. Am J Physiol Heart Circ Physiol. 2000;278(3):H971-81. doi: 10.1152/ajpheart.2000.278.3.H971. [PubMed: 10710367].

53. Hoshida S, Yamashita N, Igarashi J, Nishida M, Hori M, Kamada $\mathrm{T}$, et al. Nitric oxide synthase protects the heart against ischemia-reperfusion injury in rabbits. J Pharmacol Exp Ther. 1995;274(1):413-8. [PubMed: 7542338].

54. Gurjar MV, Sharma RV, Bhalla RC. eNOS gene transfer inhibits smooth muscle cell migration and MMP-2 and MMP-9 activity. Arterioscler Thromb Vasc Biol. 1999;19(12):2871-7. doi: 10.1161/01.atv.19.12.2871. [PubMed: 10591663].

55. Wang D, Yu X, Brecher P. Nitric oxide and N-acetylcysteine inhibit the activation of mitogen-activated protein kinases by angiotensin II in rat cardiac fibroblasts. J Biol Chem. 1998;273(49):33027-34. doi: 10.1074/jbc.273.49.33027. [PubMed: 9830056].

56. Locatelli V, Rossoni G, Schweiger F, Torsello A, De Gennaro Colonna V, Bernareggi M, et al. Growth hormone-independent cardioprotective effects of hexarelin in the rat. Endocrinology. 1999;140(9):4024-31. doi: 10.1210/endo.140.9.6948. [PubMed: 10465272].

57. Weber KT. Extracellular matrix remodeling in heart failure: a role for de novo angiotensin II generation. Circulation. 1997;96(11):4065-82. doi: 10.1161/01.cir.96.11.4065. [PubMed: 9403633].

58. Spinale FG, Coker ML, Heung LJ, Bond BR, Gunasinghe HR, Etoh $\mathrm{T}$, et al. A matrix metalloproteinase induction/activation system exists in the human left ventricular myocardium and is upregulated in heart failure. Circulation. 2000;102(16):19449. doi: 10.1161/01.cir.102.16.1944. [PubMed: 11034943].

59. Ruiz-Ortega M, Rodríguez-Vita J, Sanchez-Lopez E, Carvajal G, Egido J. TGF- $\beta$ signaling in vascular fibrosis. Cardiovasc Res. 2007;74(2):196-206. doi: 10.1016/j.cardiores.2007.02.008. [PubMed: 17376414].

60. Luo J, Gao X, Peng L, Sun H, Dai G. Effects of hydrochlorothiazide on cardiac remodeling in a rat model of myocardial infarctioninduced congestive heart failure. Eur J Pharmacol. 2011;667 (1-3):314-21. doi: 10.1016/j.ejphar.2011.06.012. [PubMed: 21703256].

61. Jneid H, Alam M, Virani SS, Bozkurt B. Redefining myocardial infarction: what is new in the ESC/ACCF/AHA/WHF Third Universal Definition of myocardial infarction? Methodist Debakey Cardiovasc J. 2013;9(3):169-72. doi: 10.14797/mdcj9-3-169. [PubMed: 24066201].

62. Soriano FG, Guido MC, Barbeiro HV, Caldini EG, Lorigados CB, Nogueira AC. Endotoxemic myocardial dysfunction: subendocardial collagen deposition related to coronary driving pressure. Shock. 2014;42(5):472-9. doi: 10.1097/SHK. 0000000000000232. [PubMed: 25051283].

63. Kim NN, Villegas S, Summerour SR, Villarreal FJ. Regulation of cardiac fibroblast extracellular matrix production by bradykinin and nitric oxide. J Mol Cell Cardiol. 1999;31(2):45766. doi: 10.1006/jmcc.1998.0887. [PubMed: 10093057].

64. Janssens S, Pokreisz P, Schoonjans L, Pellens M, Vermeersch P, Tjwa M, et al. Cardiomyocyte-specific overexpression of nitric oxide synthase 3 improves left ventricular performance and reduces compensatory hypertrophy after myocardial infarction. Circ Res. 2004;94(9):1256-62. doi: 10.1161/ 01.RES.0000126497.38281.23. [PubMed: 15044322].

65. Smith RSJr, Agata J, Xia CF, Chao L, Chao J. Human endothelial nitric oxide synthase gene delivery protects against cardiac remodeling and reduces oxidative stress after myocardial infarction. Life Sci. 2005;76(21):2457-71. doi: 10.1016/j.lfs. 2004.11.028. [PubMed: 15763077].

66. Prabhu SD. Nitric oxide protects against pathological ventricular remodeling: reconsideration of the role of NO in the failing heart. Circ Res. 2004;94(9):1155-7. doi: 10.1161/01.Res.0000129569.07667.89. [PubMed: 15142968].

67. Murohara T, Asahara T, Silver M, Bauters C, Masuda H, Kalka C, et al. Nitric oxide synthase modulates angiogenesis in response to tissue ischemia. J Clin Invest. 1998;101(11):2567-78. doi: 10.1172/JCI1560. [PubMed: 9616228]

68. Hu A, Jiao X, Gao E, Koch WJ, Sharifi-Azad S, Grunwald Z, et al. Chronic $\beta$-adrenergic receptor stimulation induces cardiac apoptosis and aggravates myocardial ischemia/reperfusion injury by provoking inducible nitric-oxide synthase-mediated nitrative stress. J Pharmacol Exp Ther. 2006;318(2):469-75. doi: 10.1124/jpet.106.102160. [PubMed: 16574780].

69. Kim YM, Bombeck CA, Billiar TR. Nitric oxide as a bifunctional regulator of apoptosis. Circ Res. 1999;84(3):253-6. doi: 10.1161/01.res.84.3.253. [PubMed: 10024298].

70. Kim YM, Kim TH, Seol DW, Talanian RV, Billiar TR. Nitric oxide 
suppression of apoptosis occurs in association with an inhibition of Bcl-2 cleavage and cytochrome crelease. J Biol Chem. 1998; 273(47):31437-41. doi: 10.1074/jbc.273.47.31437. [PubMed: 9813055].

71. Pang JJ, Xu RK, Xu XB, Cao JM, Ni C, Zhu WL, et al. Hexarelin protects rat cardiomyocytes from angiotensin II-induced apoptosis in vitro. Am J Physiol Heart Circ Physiol. 2004;286(3):H1063-9. doi: 10.1152/ajpheart.00648.2003. [PubMed: 14615277]. 